\title{
FORMACIÓN DE COMPETENCIAS EMPRESARIALES PARA UN FUTURO LÍDER EN EL CONTEXTO DE UNA CLASE DE INGLÉS, EN EDUCACIÓN MEDIA VOCACIONAL
}

\section{BUSINESS SKILLS TRAINING FOR A FUTURE LEADER IN AN ENGLISH CLASS CONTEXT, IN VOCATIONAL SECONDARY EDUCATION}

\section{Sergio Armando Ramírez Infante ${ }^{1}$}

\section{Astrid Ramírez Valencia ${ }^{2}$}

UDFJC

\section{RESUMEN}

Este artículo reflexiona sobre la necesidad de formar jóvenes emprendedores en las instituciones escolares públicas o privadas en nuestra nación, sobre todos a los que cursan la educación media vocacional, para que estén

1 Estudiante en formación del programa de licenciatura en Educación Básica con Énfasis en inglés, Universidad Distrital Francisco José de Caldas, Docente de inglés en diferentes instituciones educativas de primaria y secundaria, privadas. saramirezi@correo.udistrital.edu.co. Correo ORCID https://orcid.org/0000-0003-0563-8006

2 Docente Universidad Distrital Francisco José de Caldas Bogotá, Colombia, Ph.D. en Lenguaje y cultura, formadora de profesores de inglés durante más de 25 años. Investigadora y maestra en la Universidad Distrital Francisco José de Caldas. correo aramirezv@udistrital.edu.co ORCID: https://orcid.org/0000-0002-3025-5982 en capacidad de encontrar soluciones a las problemáticas regionales, en concordancia con las políticas de la globalización. Este artículo se sustenta en las políticas que, sobre competencias para emprendedores, han sido emanadas por el Ministerio de Educación Nacional, y busca enfatizar la necesidad de potenciar el aprendizaje de la lengua extranjera inglesa por las múltiples posibilidades de conexión ofrecidas a quien domina este idioma, en los tiempos de la globalización.

Esta afirmación permite concluir que la escuela regional debe direccionar sus procesos formativos en los jóvenes de educación media vocacional hacia el desarrollo de habilidades que 
le permita interactuar en el mundo productivo, generando criterios que le sirvan de carta de navegación para educar los líderes del futuro de esta nación.

PALABRAS CLAVE: empoderamiento, competencia, liderazgo, inglés

\section{ABSTRACT}

This article reflects on the need to train young entrepreneurs in public or private school institutions in our nation, especially those who are studying secondary vocational education, so that they are able to find solutions to the regional problems, according to the globalization policies. This article is based on the policies that, on competences for entrepreneurs, have been established by the Ministry of National Education, and seeks to emphasize the need to promote the learning of the English as a foreign language by the multiple possibilities of connection offered to those who master this language, in the globalization times.

This affirmation allows us to conclude that the regional school must direct its formative processes in the young people of vocational secondary education towards the development of abilities that allow them to interact in the productive world, generating criteria that serve as a navigation chart to educate the future leaders in this nation.

KEYWORDS: empowerment, competence, leadership, English

\section{INTRODUCCIÓN}

La globalización ha tenido incidencia en la creación de empresas trayendo múltiples alcances frente al surgimiento de este fenómeno, en este sentido, habrá que reconocer su incidencia en el campo educativo, por ser el promotor de acciones visionarias en una sociedad que con el uso de la tecnología y la capacitación del recurso humano podrá estar acorde a las necesidades del mundo contemporáneo.

A causa de ello, esta era permite la integración de la economía, lo cual involucra cambios frente a la generación de nuevos mercados atractivos en diversos contextos y que promueven la inversión del capital, entrándose en el mundo de un comercio que está al alcance de quien decida hacer una apuesta emprendedora, que integre lo humano y lo comercial.

Esta situación ha llevado al surgimiento de nuevas ideas y al florecimiento de proyectos de emprendimiento, entendido por Rubio (2007) cómo "aprender a detectar las oportunidades que ofrece el entorno"( p. 74); en otras palabras, este autor da visos de las otras posibilidades que puede brindar la escuela al contexto global, lo cual implica la generación de espacios dentro del plan de estudios de los colegios, que hagan posible esta proyección desde los primeros años de escolaridad, sin dejar de lado, los valores formativos, tan necesarios, para el joven de estos tiempos.

Adviértase, en efecto, que la globalización le plantea a la escuela retos para que el joven vaya más allá de las fronteras de su país y esté en capacidad de desenvolverse para participar en la economía global, de tal forma que se fortalezca su capacidad de compromiso y cambio, motivándolo a alcanzar objetivos a pesar de las múltiples dificultades u obstáculos que pueda tener para lograrlos, de este modo, deberá buscar soluciones a aquellas problemáticas identificadas tanto en la escuela, como en su entorno, o en su familia, asumiendo una actitud creativa en la búsqueda de soluciones viables y posibles, actuando como un ciudadano de la era globalizante.

Al respecto conviene decir que pensar en el mundo globalizado, lleva necesariamente a cambiar los criterios de formación en términos de competencias empresariales las cuales se 
han venido desarrollando en este bachiller, especialmente por las nuevas situaciones de la sociedad cambiante, marcada por la conectividad y la innovación tecnológica.

En consecuencia, la educación del momento debe estar acorde a las demandas y exigencias requeridas por el contexto laboral actual, aceptada esta condición, vale la pena mencionar a Trilling \& Fadel (2009) quienes proponen el desarrollo de la habilidad y la innovación; para ello, sugieren ofrecer un conocimiento digital, así como implementar unas habilidades para la vida y el desempeño ocupacional.

Agregado a esto, se demanda tener la habilidad comunicativa, el trabajo en equipo, el pensamiento crítico e innovador, la capacidad para resolver problemas, la adaptabilidad, la dirección y la responsabilidad, ejes importantes para impulsar el desempeño del joven como ciudadano global, requiriendo además la resiliencia en el desarrollo de cualquier trabajo u ocupación.

Ahondemos todavía un poco más para decir que, la escuela debe brindar ambientes propicios para la comunicación asertiva, concertadora y colaborativa, con miras a proponer soluciones a las dinámicas que allí se viven, para potencializar el aprendizaje en contextos para este caso, el empresarial o gerencial.

Al llegar aquí es oportuno hacer referencia a los lineamientos planteados por el MEN (2007) en el abordaje de "las competencias laborales" definidas como el conocimiento, habilidades, actitudes y valores que capacitan al joven para desempeñarse en un entorno productivo, sin tener en cuenta, tanto el tipo de cargo, así como la actividad desarrollada, ni la responsabilidad solicitada para el desempeño de una labor, en cualquier sector económico.

En esta perspectiva, la entidad gubernamental previamente mencionada plantea una serie de consideraciones que pretenden potencializar las habilidades en los jóvenes, que le permitan entrar en un proceso de inmersión, en cualquier sector productivo, nacional o internacional.

La ilustración que aparece a continuación, deja ver las habilidades que la escuela deberá desarrollar en los jóvenes, para alcanzar una articulación con el sector productivo, económico y social.

\section{HABILIDADES PARA EL MUNDO PRODUCTIVO (MEN)}
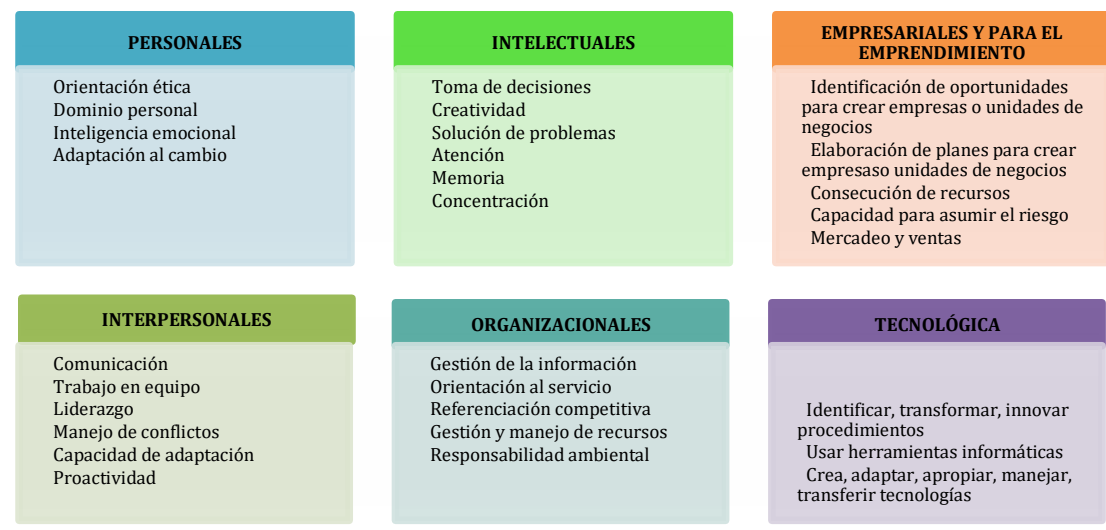

Figura 1. Habilidades para el mundo productivo Fuente: MEN. (2007). Elaboración Propia 
La figura 1 incluye seis macro competencias entre las cuales se encuentra: la competencia personal relacionada con los comportamientos y actitudes que se espera sean alcanzados dentro del contexto productivo, económico y social, desde una perspectiva ético, personal y inteligencia emocional, demostrando flexibilidad frente al cambio.

Agregado a lo anterior, está la competencia intelectual que da cuenta del proceso de pensamiento en relación con la toma de decisiones, la creatividad, la solución de problemas, la atención, la memoria y la concentración.

Por otro lado, la competencia empresarial incluye el empoderamiento para que el joven organice, lidere y sostenga un negocio bajo su iniciativa; en tal sentido, se espera que esté en capacidad de crear planes, conseguir recursos y asumir riesgos, que le permitan forjar acciones de mercadeo y ventas, proyectándolas a su vida personal, emocional y social.

Así mismo, la competencia interpersonal se enmarca en la capacidad de adaptarse a los entornos laborales y sociales, de tal manera que pueda interactuar coordinadamente con otros, para lo cual requiere de una habilidad comunicativa, de trabajo en equipo, liderazgo, manejo de conflictos, mostrando adaptabilidad, y siendo proactivo, cuando interactúa con otros miembros de un grupo.

Al lado de, ello está la competencia organizacional que le da la capacidad de aprender cuando interactúa con otros, haciendo uso de un pensamiento estratégico, con una aptitud de manejo de la gestión de la información, orientado al servicio, la referencia competitiva, la gestión y la administración de recursos, con una responsabilidad ambiental y social.

Por último, está la competencia tecnológica que le permite identificar, transformar e innovar los procedimientos, los métodos y los artefactos a través del dominio de herramientas informáticas, según sea su alcance, llevándolo al mismo tiempo, a la elaboración de nuevos modelos tecnológicos.

Esta idea se refuerza con Barrios (2009) quien explica la importancia de las competencias laborales por posibilitar la inclusión a la vida tanto laboral, social como económica, en el momento en que una persona desea incorporarse dentro de cualquier contexto ocupacional, social o productivo.

De esta forma se potenciarán las ideas, pero ante todo se fortalecería su capacidad de compromiso y entrega del joven, con los acontecimientos y hechos de su cotidianidad nacional e internacional, esperando que esté en capacidad de liderar proyectos personales y laborales con un alto grado de compromiso y viabilidad.

Al llegar a este punto, se pretende que actúe asertivamente, sepa trabajar en equipo, teniendo un sentido ético para manejar recursos económicos y financieros, tanto a nivel personal como empresarial, con capacidad para ver los problemas como oportunidades y aprendiendo de las experiencias de otros. Asimismo, se espera que adquieran las bases para crear, liderar y sostener negocios por cuenta propia, o aportar e innovar en transacciones nacionales o internacionales.

Puede afirmarse que, con el dominio de estas competencias, un estudiante que culmine su educación media en el siglo XXI, conseguiría desarrollar una inteligencia práctica y una mentalidad emprendedora para la vida productiva, e incluso para actuar en otros ámbitos globales.

Para viabilizar lo anteriormente planteado, es importante tener en cuenta algunas consideraciones relacionadas con el tema 
del emprendimiento, en el contexto educativo escolar, las cuales serán esbozadas a continuación.

\section{EL EMPRENDIMIENTO}

Hablar de emprendimiento conduce a señalar algunas definiciones, Valls, N. y otros (2009), la conceptualiza como "la capacidad de iniciar, crear y poner en marcha un proyecto, a través de la identificación de ideas y oportunidades, analizando los factores exógenos (económicos, sociales, ambientales y políticos) así como los endógenos (capacidad de disponer de personas, así como de recursos físicos y financieros)".

En esta definición se resalta la consolidación de las ideas en proyectos que convierten los problemas en oportunidades determinadas por factores externos e internos por parte del emprendedor.

Por otro lado, Pereira (2003) lo define en términos de identificación de oportunidades que llevan a la creación de una organización o plan, con el propósito de ser aprovechadas y convertirse en potencialidades, en esta perspectiva se resalta la agudeza que tiene el emprendedor para identificar aquellas situaciones que en un momento dado probablemente pueden ser concebidas como dificultades, pero que se convierten en oportunidades para desarrollar ideas, que permitan plantear soluciones.

Con este panorama es posible establecer una relación entre el emprendimiento y la educación, al respecto, Gibb (2005) expone que la educación en emprendimiento requiere de una integración del conocimiento de varias disciplinas, además de mayores oportunidades de espacios de aprendizaje, a través de la experiencia que permitan evaluar conocimientos específicos en la práctica, que den mayor tiempo para la reflexión, aprender haciendo, más que escuchando o leyendo, pues el proceso de fortalecimiento se da desde la praxis y desde la necesidad de articular cuidadosamente, los insumos cognitivos de los estudiantes, con una pedagogía idónea y práctica.

Esta mirada indica que en el contexto educativo es necesario generar un diálogo de saberes interdisciplinares, que potencialicen la formación de un sujeto emprendedor, el cual debe estar directamente articulado con la realidad, para tener la oportunidad de llevar a la práctica ideas innovadoras o transformadoras.

Todo esto lleva a plantear que el tema del emprendimiento debe ser incluido en la escuela, por considerarse un pilar fundamental dentro de los principios orientadores de la formación del educando del siglo XXI.

Una vez señalada esta distinción, conviene advertir que este aspecto debe ser considerado desde los primeros años escolares, de tal manera que se tracen objetivos de hacia una actitud de emprendimiento, por ser pieza primordial que permita a los jóvenes llegar a su proceso de formación media vocacional, y se pueda evidenciar en un actuar proactivo al momento en que se le presente cualquier situación dentro de su vida, ya sea personal o profesional, materializándolo en la formulación de proyectos o alternativas.

Conceptualizando el término emprendimiento, la Ley 1014 de 2006, art. $1^{\circ}$ lo concibe como una forma de pensar, razonar y actuar centrada en las oportunidades, planteadas con visión global y llevadas a cabo mediante un liderazgo equilibrado, manifestado en la gestión de un riesgo calculado, dentro del cual su resultado es la creación de un valor que beneficia a la empresa, la economía y la sociedad.

De aquí se puede percatar que un emprendedor estará en capacidad de reconocer oportunidades en los problemas o en aquellas situaciones que otros no están en capacidad de detectar, analizando los recursos disponibles, 
identificando factores de riesgo, de tal manera que trace objetivos alcanzables y seguros.

Se puede entonces afirmar que el sef emprendedor no solamente se contextualiza en el mundo de los negocios, tal y como lo plantea Núñez y otros (2016) sino por el contrario, es una condición que debe ser alcanzada por aquellos profesionales o no que siempre están en la búsqueda de soluciones innovadoras, con un pensamiento creativo frente a las dificultades, que potencializan su capacidad de innovación y de resolución de problemas, de tal manera que estas situaciones despiertan el deseo de gestionar acciones ya sea en un marco social, personal o económico.

Bajo estas premisas cabe detallar que en la educación pública existe la tendencia por parte de los jóvenes a mostrar una actitud introspectiva y de carencia afectiva, social, económica ahondando en su baja autoestima, que en estos tiempos de pandemia han dejado ver la brecha de inequidad social que padecen, por lo tanto, propuestas como esta daría una oportunidad a muchos de ellos que gozan de talento pero les falta apropiarse de una actitud emprendedora que les permita subsanar tales brechas, para que estas circunstancias se conviertan en oportunidades, utilizando la creación, la innovación y la apuesta de acciones o actitudes que harán la diferencia dentro de su círculo social y al interior de su comunidad para así lograr trascender a otros contextos globales.

Se debe añadir que la condición innovadora depende de la particularidad de cada ser, Paños y otros (2017) enfatizan que la visión de mundo es diferente en cada persona y región, así como sus pensamientos, creencias y vivencias, por lo tanto, los aportes y los desafíos emprendedores son distintos pues dependen del contexto al cual este pertenezca.

Al llegar aquí se debe pensar en los diversos escenarios escolares que permiten promover el emprendimiento, a continuación, se muestra una apuesta dada por el MEN (2013) sobre esta temática.

\section{ESCENARIOS INSTITUCIONALES PROMOTORES DEL EMPRENDIMIENTO}

En el espacio de la escuela existe infinidad de actividades que podrían ser aprovechadas para la consolidación del emprendimiento, en tal sentido, es necesario entrar a tomar en cuenta las consideraciones planteadas por el MEN (2013) que plantea algunas acciones que pueden ser desarrolladas tanto al interior de la escuela, como dentro del contexto en que está involucrado el joven, por ser espacios promotores de la participación y del fomento de acciones emprendedoras, de quienes hacen parte de la escuela. En la gráfica 2 se presentan estos escenarios.

\section{ESPACIOS PROMOTORAS DE EMPRENDIMIENTO}

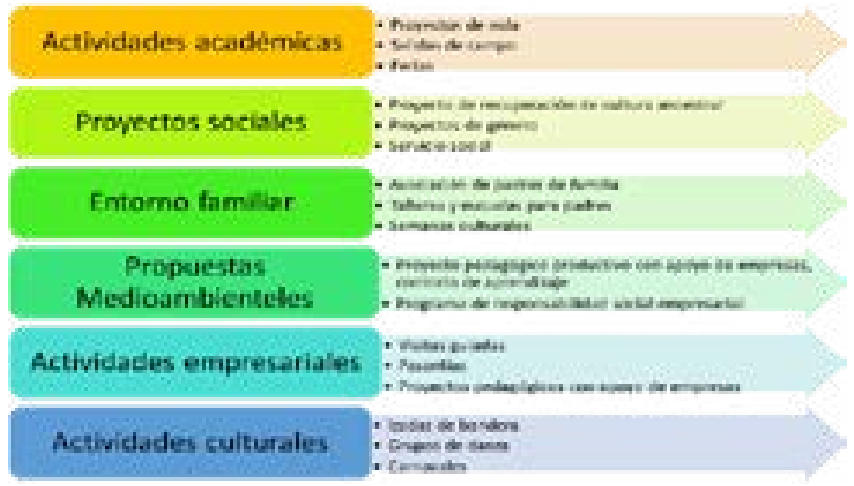

Gráfica 2. Espacios institucionales promotores del emprendimiento. Fuente: MEN (2011) Elaboración propia. 
Bien pareciera por todo lo anterior, que la escuela tiene la necesidad de poner en marcha diversas estrategias que busquen la promoción de acciones de emprendimiento enmarcadas en un aprendizaje vivencial dentro del cual, se tenga la oportunidad de entrar en un contexto real suscitador de habilidades reflexivas y de aplicación directa que lleven a otros modos de aprendizaje, que propicien el florecimiento de su condición de líder, mediante las experiencias de la vida real y cotidiana de la escuela, que lo lleven a estar inmerso en situaciones de emprendimiento.

Bien vistas las cosas, se puede detectar que la escuela incluye múltiples escenarios que hacen parte las actividades académicas, tal es el caso de las propuestas de mejoramiento del entorno medioambiental, las actividades empresariales y las culturales siendo estos espacios promotores del trabajo mancomunado y cooperativo, que tratan muchas veces problemáticas de su comunidad y de la escuela, que lo llevan a empoderarse de las situaciones demostrando así su sentido de pertenencia y posibilitando al mismo tiempo entrar en contacto con la realidad, a través de simulaciones o situaciones que lo aquejan directa o indirectamente y que le exigen alternativas de solución viables y alcanzables.

De aquí se desprende la relevancia del trabajo en equipo, por hacer parte del entrenamiento de los emprendedores, además de atenuar un aprendizaje mutuo entre los integrantes, basado en la responsabilidad tanto personal como grupal, la interacción y el fortalecimiento de las habilidades sociales y finalmente, la capacidad de aprender de manera recíproca e interactiva.

Todo este tipo de acciones buscan generar una cultura de empoderamiento, que despierte actitudes y capacidades emprendedoras que beneficien a la comunidad a la cual pertenece el joven, pero que a su vez forje acciones que resuelvan las dificultades del contexto particular y global.

\section{EL EMPRENDIMIENTO Y LA COMPETENCIA DEL INGLÉS EN EL CONTEXTO EDUCATIVO}

Es innegable la influencia del idioma inglés en el desarrollo de las actividades de emprendimiento por ser un lenguaje universal, atribución evidenciada en varios estudios, entre los cuales se encuentra el del MEN (2014, Dialogo Interamericano, 2017), que poco reconoce el impacto de la lengua inglesa en la competitividad internacional, que aunado al fenómeno de la globalización, ha dinamizado las relaciones comerciales entre diversos países, reconociéndose así tal como lo plantea Jiménez \& Parra (2008) la necesidad de dominar una lengua extranjera, por ser un instrumento de oportunidad y emprendimiento en para los jóvenes que tienen una competencia superior.

A causa de ello, quien maneje una lengua extranjera (inglés) potencia sus habilidades tanto personal como laboral, frente a las nuevas condiciones del mercado global, porque esta lengua se ha convertido en un medio de acceso y comunicación, favorecedor de múltiples prácticas de emprendimiento en diversos contextos, tanto locales como internacionales.

Abundan posturas que ratifican este argumento entre las cuales se encuentra la de Ufier (2015) quien reconoce el grado de influencia del dominio de una lengua extranjera en las relaciones comerciales especialmente con países de habla inglesa, por generar confianza y un ambiente favorable y propicio para establecer convenios, producto de relaciones tanto comerciales como culturales.

Ante esta consideración se debe aceptar que en el mundo laboral existe una marcada exigencia del dominio de una lengua extranjera (Inglés), la cual está directamente relacionada con los salarios ofrecidos por las compañías, así como con los estímulos que pueden recibir los emprendedores, aunado a la condición de que muchas de las instrucciones relacionadas 
con el uso de diversos artefactos y de acceso a la tecnología están escritos en lengua inglesa, lo cual obliga a que los emprendedores sean competentes en este idioma.

Al llegar aquí tenemos que Melitz (2008) explica cómo las oportunidades de emprendimiento se relacionan con las inversiones provenientes de países extranjeros, que para el caso colombiano, hay muchos interesados en invertir, pero una de las barreras que los frena, es la falta de dominio del idioma inglés para entablar contacto directo con dichas empresas por parte de los emprendedores Colombianos, hecho que los restringe, reduciéndose así la posibilidad que tienen estos emprendedores, por sentirse intimidados al no poder promocionar sus ideas y propuestas en el idioma extranjero directamente, sino que muchas veces deben acudir a intermediarios.

Se recurre a este argumento para exponer la necesidad de reconocer que la competencia lingüística del inglés hace parte de los conocimientos que deben manejar un emprendedor, especialmente por las oportunidades generadas para quienes manejan una lengua extranjera, por brindarles la opción de proyectar ideas innovadoras en el campo social, político, cultural, étnico y económico, así como en el de la ciencia y la tecnología, fortalecedoras del desarrollo de las habilidades comunicativas y las competencias de emprendimiento.

Adviértase que, a pesar de lo valioso que resultan las consideraciones del MEN (2014) respecto a las competencias que deben dominar un joven emprendedor, no toma en cuenta que el dominio de la lengua extranjera también hace parte estratégica e imprescindible, para acceder al mundo de los negocios.

Ahora resulta cierto que este conocimiento del idioma le permite interactuar en un contexto globalizado promotor de ideas innovadoras y de una comunicación permanente entre múltiples agentes, para alcanzar logros, en la implementación y el desarrollo de sus proposiciones e ideas.

Análogamente, la coyuntura de crecimiento ofrecida por el dominio de una lengua extranjera está acompañada de la apertura de nuevas eventualidades que fortalecen la autoestima de los emprendedores, lo cual implica no solamente conocer el idioma, sino tener un desempeño oral asertivo, especialmente por hacerlo competitivo para concertar, convencer y promover ideas que generarían diversos negocios, pues no es lo mismo usar un intérprete, que ser un interlocutor comercial directo, lo cual amerita la necesidad de acompañar los procesos de aprendizaje de una lengua extranjera, con el uso de herramientas tecnológicas en la escuela, para que se tenga acceso a las aplicaciones facilitadoras del desarrollo de la habilidad oral de la lengua, de esta manera se subsanaría la desigualdad en la brecha digital y del idioma en pos de un proceso de expansión y mejoramiento de las actitudes de los emprendedores, dándoles un valor agregado a las ideas que tengan por desarrollar e implementar.

\section{SUGERENCIAS PARA FORMAR ESTUDIANTES EMPRENDEDORES DESDE LA CLASE DE INGLÉS}

Hablar de emprendimiento se traduce en la pesquisa de los conocimientos requeridos por los jóvenes de hoy, en esta sección se plantea una propuesta de formación de jóvenes emprendedores que curse educación media, en instituciones públicas o privadas; para alcanzar este propósito se debe tomar las consideraciones presentadas a lo largo de este artículo, por lo cual resulta pertinente considerar los lineamientos de entes gubernamentales como el MEN, para dar respuesta a las problemáticas y políticas de la globalización en nuestro país, por su impacto en el mundo actual. 
En concordancia con esta perspectiva, se requiere de la toma de conciencia de los docentes en ejercicio frente a la necesidad de generar cambios en la implementación del campo relacionado, para este caso con la enseñanza del idioma, de tal forma que se optimice la oralidad de los jóvenes emprendedores, enfocándose en el desarrollo de la capacidad comunicativa del inglés, por ser este un requisito de la sociedad global que afecta directamente el ámbito comercial, de quienes tienen ideas innovadoras, por lo cual estos jóvenes están corriendo el riesgo de perder oportunidades de negocios o académicas, afectando así su desarrollo empresarial o personal.

Adviértase que en esta era tecnológica, las redes sociales transversalizan todos los campos de estudio, situación que ha llevado a convertir el idioma inglés en un eje fundamental para permitirle interactuar en el mundo digital, sin embargo, es preciso reconocer que este conocimiento debe ir no solo por el dominio de la lengua, sino que debe trascender hacia la formación de jóvenes emprendedores, por lo tanto, el área de la lengua extranjera no puede seguir siendo solamente una asignatura que desarrolle contenidos, sino por el contrario, este objetivo debe convertirse en un proyecto de vida, que involucre el conocimiento de diversos temas que son relevantes y que posibilitan la implementación de proyectos de emprendimiento, de corto o largo alcance.

De aquí se desprende la visión multidisciplinar que requiere el área de inglés, por ser un espacio de conexión de diversos conocimientos, en este caso, los relacionados con el dominio de la lengua, y con la innovación, el emprendimiento y la creación, que posibilitan el desarrollo de las competencias personales, intelectuales, empresariales, interpersonales, organizacionales y tecnológicas. por brindar alternativas para el desarrollo y la puesta en marcha de proyectos emprendedores, situación que simultáneamente lo direcciona hacia un liderazgo, al ser copartícipe del trabajo en equipo.

Con todo lo anterior, se evidencia la necesidad de diseñar diversas alternativas de formación de jóvenes emprendedores, con las cuales sea posible la resolución de los problemas que viven dentro de su contexto local y global, pues si se ajusta su proceso de formación en lo que respecta al dominio de la lengua extranjera en tiempos de globalización, acompañado del uso de la tecnología, se tendrá la posibilidad de poner en marcha acciones emprendedores, acompañadas de un pensamiento crítico que propendan por la conservación del medio ambiente, el respeto por el otro, con capacidad creativa y generadora de ideas ingeniosas, que resuelvan los problemas de su entorno, su comunidad y del mundo entero.

Al llegar a este punto, es necesario hablar sobre la importancia de implementar metodologías de la enseñanza de la lengua que sea más efectivas, que las que convencionalmente se han desarrollado, haciendo uso de las diversas aplicaciones disponibles para teléfonos inteligentes, tablets o cualquier otro medio electrónico, que propenda por el uso cotidiano del inglés, convirtiendo su aprendizaje en un objetivo que está a su alcance para luego convertirlo en un proyecto de vida.

Para ello, se recomienda trazar objetivos que sean de corto alcance, pero efectivos que le permitan comunicarse de manera rápida y estratégica, por lo cual se sugiere rodearse de personas que tengan este mismo propósito, es decir que estén interesados en aprender inglés y que de cierta forma lo comprometan y lo involucren en actos de habla, que le resulten llamativos, atractivos y facilitadores del aprendizaje del idioma. 
De igual manera, se recomienda la implementación de metodologías de la enseñanza de la lengua que promuevan el autoaprendizaje y la autoconfianza por parte de quien aprende la lengua, de tal manera que asuma el riesgo de hablar sin temor a equivocarse, pues es necesario reconocer que el error en las situaciones de habla, son manifestaciones de los procesos de aprendizaje del idioma, al mismo tiempo, se sugiere que el joven emprendedor identifica su manera particular de aprender el idioma, de este modo, se convertirá en parte activa de su propio aprendizaje.

Para cerrar la idea, conviene dar a conocer la experiencia que he tenido como docente de inglés y formador de jóvenes emprendedores en una institución privada, media vocacional, en un colegio localizado en Ubaté, Boyacá, Colombia, espacio en el cual se sugirió a los estudiantes diseñar propuestas de emprendimiento que en un comienzo resultaron ser poco innovadores y descontextualizadas de las necesidades y temáticas globales, así como carentes del apoyo tecnológico.

Frente a esta situación se indagó sobre las posibles razones de este hecho, definiéndose que probablemente por su ubicación geográfica, sus costumbres, su cultura y la escasa o nula necesidad de curiosear otros mundos, hacian que sus apuestas emprendedoras estuvieran alejadas de las situaciones mundiales, así como de los hábitos de consumo globales fácilmente detectables, una vez se pone en marcha la utilización de herramientas tecnológicas y del internet, para definir necesidades que va más allá del universo local, en el cual se encuentra inmerso el joven aprendiz, notándose un cambio en cuanto a la curiosidad, pero a la vez una gran dificultad, por no poder entender los textos discursivos que están en las redes en el idioma inglés, hecho que evidencia la importancia de esta propuesta, que busca subsanar esta gran dificultad en los departamentos de Colombia.

En resumen, lo expuesto hasta aquí pretende dar respuesta a las inquietudes planteadas mediante la presentación de una propuesta, dentro de la cual se requiere impartir la enseñanza de la lengua apoyada en las herramientas tecnológicas, aprovechando el uso de los dispositivos inteligentes, que así como viabilizan el acceso a las redes sociales, también posibilitan el aprendizaje de la lengua, de tal forma que se trascienda las fronteras locales y se involucre en el mundo global, para finalmente, llevar al joven aprendiz a formular proyectos de emprendimiento, acordes a las dinámicas de la actualidad.

\section{CONCLUSIONES}

La tarea de apostar por un cambio en el aprendizaje de la lengua inglesa que busque afianzar el emprendimiento a nivel personal y social, permite plantear que este es el momento del cambio, las circunstancias del momento dejan ver que la tecnología se ha convertido en el brazo motor de interrelación en la sociedad, y por ende no se puede desconocer que el idioma inglés ha cobrado reconocimiento, por haberse convertido en el lazo facilitador de la comunicación entre diversas comunidades del mundo global.

Al llegar aquí, es necesario apreciar que esta indagación dejó ver que tanto el inglés, como el español pueden ser consideradas lenguas universales, pues a partir de la globalización se han trascendido las fronteras físicas entre los países, para entrar a interactuar en la bien llamada aldea global, que requiere de un lenguaje común.

De otro lado, no se puede desconocer la responsabilidad que le cabe a la escuela para emprender acciones pedagógicas distintas a las tradicionalmente desarrolladas, que en su 
momento fueron pertinentes, pero que en la actualidad deben propender por implementar un pensamiento emprendedor, creativo y conectado con la realidad, tanto local como global del joven, para entrar en nuevas dinámicas de interacción virtual, acordes con las políticas del momento, de allí la necesidad de crear una escuela que adopte una mirada global con incidencia en lo local, pero siempre rescatando lo cultural, sin dejarse influenciar por aquellos factores externos que están ocurriendo en el planeta, sino por el contrario, conservando y dignificando la riqueza cultural y su idiosincrasia, de ahí la trascendencia de los jóvenes emprendedores regionales.

Con todo esto en mente algunas propuestas de mejoramiento relacionadas con la formación de jóvenes emprendedores, serían las siguientes:

1. La educación media debe apasionarse por generar diversas estrategias que propicien el manejo del inglés en situaciones reales, en la perspectiva del emprendimiento.

2. La globalización plantea una nueva actitud para los jóvenes educandos y para la escuela que debe combinar la curiosidad por el mundo que lo rodea y una disposición a desarrollar capacidades y habilidades, para participar como emprendedor.

La llegada de Internet ha supuesto un vuelco irreversible en el modo en que nos comunicamos e informamos. No hay que perder de vista que un alto porcentaje de las páginas web están escritas en inglés, lo que se extiende a herramientas, recursos y demás servicios sólo disponibles en este idioma. Por ello, es una necesidad para los emprendedores tener la habilidad de comunicarse, a través de este medio en inglés y así podrá sacar partido, al enorme cúmulo de información que se encuentra en la red.

3. El Ministerio de Educación (MEN) cuando esboza las competencias requeridas por un joven emprendedor, no contempla como competencia primordial el dominio de la lengua inglesa, siendo este un factor determinante. para la comunicación que debe tener el emprendedor, por haberse convertido en una habilidad fundamental en la gestión y promoción, de ideas y propuestas.

4. Las formas de enseñar el idioma inglés para emprendedores está en mora de cambiar frente a las exigencias del mundo globalización, en consecuencia, se deberían hacer apuestas pedagógicas innovadoras y diferentes a las que se han impulsado hasta el momento, esto por la lenta efectividad que han tenido en lograr el dominio del idioma, a pesar de que el estado colombiano ha impulsado estrategias para conseguirlo, han sido poco efectivas, por la escasa conectividad a nivel regional, así como por no involucrar el uso de dispositivos a las actividades de aprendizaje del idioma, ni emplear aplicaciones que permitan entrar en contacto directo, con el uso cotidiano de esta lengua, lo cual si se implementara en la escuela, podría despertar el interés de quien aprende un idioma extranjero.

5. Es necesario, que la escuela comience a incluir otros escenarios promotores del emprendimiento y del aprendizaje de la lengua por parte de los jóvenes, para convertirlos en oportunidades, retos vivenciales e interesantes, para el aprendizaje del idioma.

6. Con el propósito de lograr una verdadera transformación en la educación del joven emprendedor, habrá que considerar e incluir las habilidades para el mundo productivo propuestas por parte del Ministerio de Educación Nacional (MEN), en los planes de estudios de las escuelas regionales, de tal manera que se conviertan en carta de navegación, para formar emprendedores en esta nación. 
7. Es innegable que la tecnología amplía la visión del mundo global por las múltiples herramientas y posibilidades que brinda, entonces, en la medida en que el estudiante pueda descubrirlas a través del dominio de la lengua extranjera inglesa, estará en capacidad de proponer soluciones inmediatas que lo lleven al descubrimiento de su visión como emprendedor, a nivel regional o mundial.

\section{REFERENCIAS BIBLIOGRÁFICAS}

Barrio De la Puente, J. (2009). Hacia una educación inclusiva para todos. Revista Complutense de Educación, 20(1), 13-31.

Espinosa, O. (2012). La Educación como Motor de Desarrollo Integral: La Importancia del Capital Humano en el Crecimiento Económico y Social de Largo Plazo (Education as a Comprehensive Development Motor: The Importance of Human Cap. Obtenido de SSRN Electronic Journal, 18

Gibb, A. (2005). Creating the entrepreneurial university worldwide. ¿Do we need a wholly different model of Entrepreneurship? realizada en Cali, Colombia: ponencia en la Cuarta Conferencia de Investigación en Entrepreneurship en Latinoamérica.

Jiménez, B. \&. (2008). El fenómeno bilingüe: perspectivas y tendencias (Vol. 73). Publicaciones Mathematicae.

L, N., M, N. C., \& Núñez, J. A. (2016). Noción de emprendimiento para una formación escolar en competencia emprendedora. Revista Latina de Comunicación Social, 20-30. doi:10.4185/RLCS-2016-1135I

Melitz, J. 2.-6. (2008). Melitz, J. Language and foreign trade, 52(4), 667-6.
Ministerio de Educación Nacional. (2005). Competencias Laborales Generales Articulación de la educación con el mundo productivo. Aportes para la construcción de currículos pertinentes. Bogotá, Colombia: Imprenta Nacional de Colombia.

Ministerio de Educación Nacional. (2017). (I. Nacional, Ed.) Obtenido de Disponible en http://aprende.colombiaaprende. edu.co/es/colombiabilingue.

Ministerio de Educación Nacional. CORPOEDUCACIÓN. (2004). Aprendiendo en el ámbito laboral. Orientaciones para el desarrollo de prácticas, observaciones y espacios pedagógicos empresariales para la formación en competencias laborales. Documento de trabajo. Bogotá, Colombia: Imprenta Nacional.

Ministerio de Educación Nacional. (5 de agosto de 1994). http://www.mineducacion.gov:co.

Ministerio de Educación Nacional. (2003). Articulación de la educación con el mundo

productivo. La formación en competencias laborales.

Paños, C. J. (2017). Educación emprendedora y metodologías activas para su fomento. Revista Electrónica Interuniversitaria de Formación del Profesorado., 20(3), 3348. Recuperado el 8 de enero de 2021, de https://www.redalyc.org/articulo. oa? $\mathrm{id}=2170 / 217052050003$

Pereira, F. (2003). Reflexión sobre algunas características del espíritu emprendedor colombiano. Colombia: Economía, Gestión y Desarrollo, 1. 
Rubio, A. (2007). Emprendimiento y creación de empresas. Bogotá: Universidad EAN.

Senado. (27 de enero de 2006). LEY 1014 Diario Oficial. Obtenido de recuperado de http://www.secretariasenado.gov.co/ senado

Trilling, B. \&. (2009). Trilling 21st century skills: Learning for life in our times. Trillian San Francisco: Trillin Jossey-Bass.

Ufier, A. (dic de 2015). The Impact of English Language Skills on National Income: A CrossNational Comparison. (44), Ufier, A. 2015 The Impact of English Language Skills on National Income: A CrossNational Comparison. Fdic, 44.

Valls, N., Villa, A., \& Martínez, S. y. (2009). Emprendimiento social Juvenil. 18 buenas prácticas. Barcelona: Fundación Bertelsmann. 